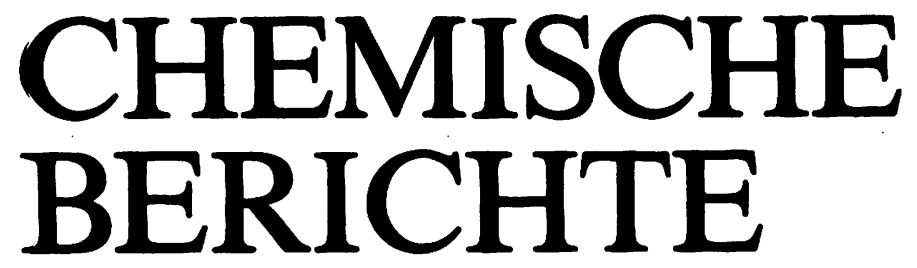

GEGRÜNDET 1868

118. JAHRGANG

HERAUSGEGEBEN IM AUFTRAG DER

GESELLSCHAFT DEUTSCHER CHEMIKER

VON

K. HAFNER - W. KIRMSE - H. MUSSO - H. NÖTH •

J. SAUER - E. WINTERFELDT

UNTER MITWIRKUNG VON

W. BECK - H. A. BRUNE - H. BUDZIKIEWICZ - W. LÜTTKE

REDAKTION: H. ZAHN

mit H. SCHILL, J. STREHLOW und A. WIELAND

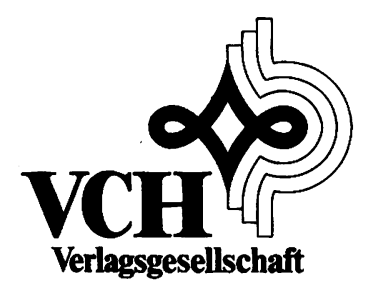




\section{INHALT VON HEFT 2}

\section{Jahrgang 1985}

\section{ANORGANISCHE CHEMIE}

Diercks Rainer und Dieck tom Heindirk: Diazadiene als Steuerliganden in der homogenen Katalyse, IX: Katalytische Cyclotetramerisierung von Propiolsäureestern ........

Beck Wolfgang, Ambach Eberhard und Nagel Ulrich: Palladium- und Platin(II)-Komplexe mit den Anionen von 6-Methyl-1,2,3-oxathiazin-4(3H)-on-2,2-dioxid und $\mathrm{N}$-2-

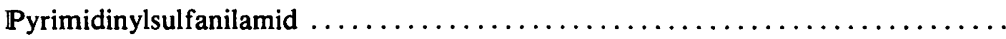

Huppmann Peter und Seppelt Konrad: Übergangsmetallverbindungen mit der Gruppe

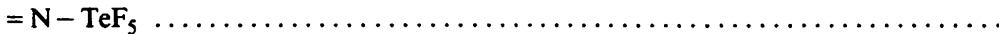

Kumpfmüller Fritz, Nölle Dieter, Nöth Heinrich, Pommerening Hans und Staudigl Rudolf: Beiträge zur Chemie des Bors, 146: Über die Reaktion von Tetrazadiborinanen mit Heterokumulenen: Pseudodipolare $[2+3]$-Cycloadditionen $\ldots \ldots \ldots \ldots \ldots$

Weber Lothar und Wewers Dietmar: Übergangsmetallkomplexe instabiler Ylide, V: Neuartige Doppelylide des Phosphors als Chelatliganden in Chromkomplexen ........

Lentz Dieter: Tetrakis(trifluormethylisocyanid)nickel, $\mathrm{Ni}\left(\mathrm{CNCF}_{3}\right)_{4}$, und Bis[ $\mu$-(trifluormethylisocyanid)-( $\eta$-cyclopentadienyl)nickel $],\left[\mathrm{Ni}\left(\mu-\mathrm{CNCF}_{3}\right)\left(\eta-\mathrm{C}_{5} \mathrm{H}_{5}\right)\right]_{2} \ldots \ldots \ldots$

Brink Klaus und Mattes Rainer: Mesylhydroxylamine, VI: Oxidation von $N$-Mesylhydroxylaminen - Schwingungsspektren und Kristallstruktur von 1,2-Dimesyl-1,2-di-

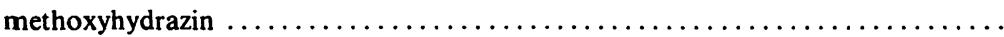

Lang Heinrich, Mohr Gerhard, Scheidsteger Olaf und Huttner Gottfried: Halogen-Metall-Austausch an komplex gebundenen Halogenphosphanen: Darstellung von

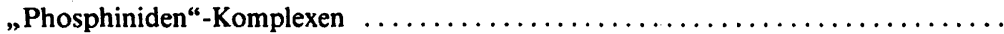

Boese Roland, Köster Roland und Yalpani Mohamed: Die Farbe von Bor-Chelaten. Röntgenstruktur-Untersuchung von Bis(4-methylphenyl)boryl- und 9-Borabicyclo[3.3.1]-

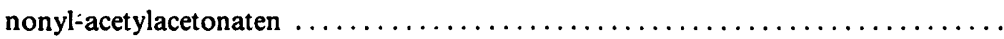

Wadepohl Hubert, Pritzkow Hans und Siebert Walter: Synthese und Struktur Platin-haltiger Tripeldecker- und Tetradecker-Komplexe $\ldots \ldots \ldots \ldots \ldots \ldots \ldots \ldots \ldots \ldots$

Appel Rolf, Knoch Falk und Zimmermann Rolf: Niederkoordinierte Phosphorverbindungen, 29: Diels-Alder-Reaktion von 2,3-Dimethylbutadien mit stabilen Phosphaalkenen als Dienophilen

\section{ORGANISCHE CHEMIE}

Lösel Walter und Daniel Helmut: 3,4-Dihydroisochinoline, I: 5,6-Dihydropyrrolo[2,1-a]isochinoline durch Umlagerung von 1-(3-Furyl)-3,4-dihydroisochinolinen ........

Diercks Rainer und Dieck tom Heindirk: Diazadiene als Steuerliganden in der homogenen Katalyse, IX: Katalytische Cyclotetramerisierung von Propiolsäureestern .......

Wamhoff Heinrich, Wald Klemens, Kirfel Armin, Farkas Lajos, Samimi Nabi und Will Georg: Reaktionen von Uracilen, 5: Verbrückte 1,2,5,6-Tetrazocane mit Uracil- und Urazolbrücken durch Dimerisierung von 5-(1,2,4-Triazolidin-1-yl)uracilen ....... 
Zarkadis Antonios K., Neumann Wilheln? P., Marr Rainer und Uzick Wolfram: Über sterisch gehinderte freie Radikale, XII: Das Radik al tert Butyldiphenylmethyl, sein Dimeres sowie deren Autoxidation. Eine Neuuntersuchung . . . . . . . . . . . . .

Kaupp Gerd und Knichala Bernd: Quantitative [1,3,2,3]-Eliminierung von Wasser aus

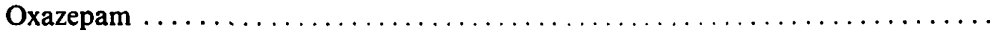

Effenberger Franz und Brodt Werner: $2(1 \mathrm{H})$-Pyridon als Austrittsgruppe bei Acylierungsreaktionen - Anwendungen in der Peptidchemie ..................

Weiske Thomas, Halim Hermann und Schwarz Helmut: C-C-Spaltungen bei ionisierten Carbonsäuren als Umkehrung der [1,4]Addition von Alkyl-Radikalen an protonierte $\alpha, \beta$-ungesättigte Carbonsäuren: Zur Rolle von Enol-Radikalkationen als reaktive Zwischenstufen bei Isomerisierungs-/Dissoziationsprozessen in der Gasphase ......

Döpp Dietrich, Krüger Carl, Makedakis George und Nour-el-Din Ahmed Moukhtar: Indoleninoxide, IX: Neuartige polycyclische linear konjugierte Cyclohexadienimine durch Umlagerung instabiler Tetrahydroisoxazolo[2,3-a] indole

Quast Helmut und Nahr Uwe: Photochemische Stickstoff-Eliminierung aus 1,4-Dihydro1-phenyl-5 $\mathrm{H}$-tetrazol-5-onen und -thionen. Benzimidazolone und Carbodiimide ....

Sohár Pál, Lázár János und Bernáth Gábor: Isolierung und Strukturaufklärung eines bei der Aminomethylierungsreaktion von $\alpha$-Methylstyrol entstehenden Nebenproduktes

Siegel Herbert, Eisenhuth Ludwig und Hopf Henning: Alkine und Cumulene, XVII: Photoadditionen von Vinylacetylen an andere ungesättigte Kohlenwasserstoffe...$\ldots$.

Anke Lutz und Weyerstahl Peter: Inter- versus intramolekulare Friedel-Crafts-Reaktion von phenylsubstituierten Dichlorcyclopropanen $\ldots \ldots \ldots \ldots \ldots \ldots \ldots \ldots \ldots$

Daub Jörg, Lüdemann Hans-Dietrich, Michna Martin und Strobl Reinhard M.: Über das korrespondierende Verhalten von heteroatom-stabilisierten Carbokationen und dem Cycloheptatrien-Norcaradien-Gleichgewicht $\ldots \ldots \ldots \ldots \ldots \ldots \ldots \ldots \ldots \ldots$

Hoffmann Reinhard W. und Barth Wolfgang: Carben-Reaktionen, XVII: Nitrilylide

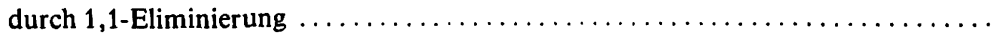

Kupfer Rainer, Würthwein Ernst-Ulrich, Nagel Michael und Allmann Rudolf: 1-Alkoxy2-azaallenium-Salze: Röntgenographische und quantenmechanische Strukturunter-

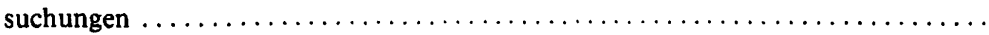

Verkoyen Carl und Rademacher Paul: Reduktion von $\beta$-Lactamen, III: Darstellung von $\beta$-Thiolactamen und Reduktion mit Raney-Nickel ...................

Yalpani Mohamed und Wilke Gilnther: Hydratisierte Oxokohlenwasserstoffe, IV: Silylierung von 1,1-Dihydroxy-Verbindungen

Boese Roland, Köster Roland und Yalpani Mohamed: Die Farbe von Bor-Chelaten. Röntgenstruktur-Untersuchung von Bis(4-methylphenyl)boryl- und 9-Borabicyclo[3.3.1]-

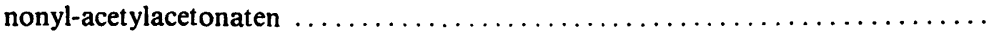

Horner Leopold und Lindel Hans: Phosphororganische Verbindungen, 111: Phosphinund Thiophosphinsäure-cyanide als fluoreszierende SH-selektive Reagenzien .....

Bäuml Englbert und Mayr Herbert: Silbertrifluoracetat-initiierte Reaktionen von Chlortriphenylallen mit Cyclopentadien - Vinylkation-analoge Cycloadditionen von Alle-

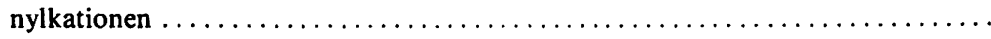


Bäuml Englbert und Mayr Herbert: Cycloadditionen des Triphenylallenyl-Kations mit Cyclopentadien - Studium des Reaktionsmechanismus unter stabilen Ionen-Bedin-

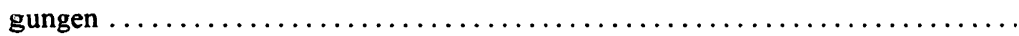

Maier Günther, Roth Cornelia und Schmitt Reinhart K.: Diastereoselektivität bei der Hydridreduktion acyclischer Diketone $(1,2-, 1,3-, 1,4-$ und 1,5-Induktion) . . . . . . . .

Maier Günther, Schmitt Reinhart K. und Seipp Ulrich: Umkehrung der Diastereoselektivität bei der Hydridreduktion acyclischer Diketone $\ldots \ldots \ldots \ldots \ldots \ldots \ldots \ldots$

Effenberger Franz, Ziegler Thomas und Schönwälder Karl-Heinz: Enolether, XVI: Syn-

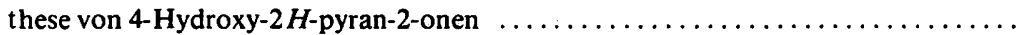

Lenoir Dieter und Frank Robert M.: Polare Effekte von 4-Substituenten bei der Solvolyse von Tetracyclo[6.2.1.1 $1^{3,6} \cdot 0^{2,7}$ ]dodecan-11-yl-triflaten. Through-space-Effekt im Vergleich zum induktiven Effekt

Bauer Walter, Laube Thomas und Seebach Dieter: Kristall- und Molekülstruktur eines mit THF solvatisierten dimeren Lithiumenolats eines Carbonsäureamids

Gotthardt Hans, Feist $\dagger$ Ulrich und Schoy-Tribbensee Sabine: Synthese und Eigenschaften

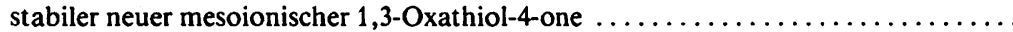

Gotthardt Hans und Feist $\uparrow$ Ulrich: Ein neuer Zugang zu Alkylthio(trifluoracetyl)furanen durch thermische [3+2]-Cycloadditionen neuer mesoionischer 1,3-Oxathiol-4-one an

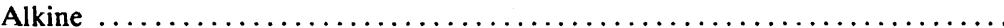

Staab Heinz A., Jörns Manfred, Krieger Claus und Rentzea Marina: Elektron-DonorAcceptor-Verbindungen, XXXVI: Chinone und Chinhydrone der [2.2]- und [3.3]Metaparacyclophan-Reihen

Eilbracht Peter, Balß Erika und Acker Michael: Zur Regio- und Stereoselektivität der hydrocarbonylierenden Cyclisierung von 1,4-Dienen mit Kohlenmonoxid: Synthese des $( \pm)$ - $(\alpha)$-Cuparenons

Hashem Md. Abul, Hülskämper Ludwig und Weyerstahl Peter: Thermolyse und Photolyse von Hexachlor-tris- $\sigma$-homotropon und verwandten Verbindungen $\ldots \ldots \ldots \ldots$

Griesbaum Karl und Meister Martin: Beweise gegen postulierte ungewöhnlich stabile Primärozonide von 1,4-Dichlor-2-butenen $\ldots \ldots \ldots \ldots \ldots \ldots \ldots \ldots \ldots \ldots \ldots \ldots \ldots \ldots$ 


\section{INORGANIC CHEMISTRY}

Diercks Rainer and Dieck tom Heindirk: Diazadienes as Controlling Ligands in Homogeneous Catalysis, IX: Catalytic Cyclotetramerization of Propynoic Esters .......

Beck Wolfgang, Ambach Eberhard, and Nagel Ulrich: Palladium and Platinum(II) Complexes with the Anions of 6-Methyl-1,2,3-oxathiazin-4(3H)-one 2,2-Dioxide and $\mathrm{N}$ -

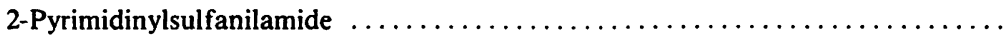

Huppmann Peter and Seppelt Konrad: Transition Metal Compounds with the Ligand

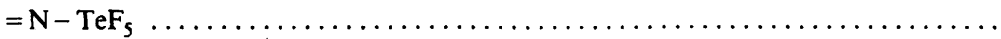

Kumpfmüller Fritz, Nölle Dieter, Nöth Heinrich, Pommerening Hans, and Staudigl Rudolf: Contributions to the Chemistry of Boron, 146: Reactions of Tetrazadiborinanes with Heterocumulenes: Pseudodipolar [2+3]-Cycloadditions

Weber Lothar and Wewers Dietmar: Transition Metal Complexes of Instable Ylides, V: Novel Double Ylides of Phosphorus as Chelating Ligands in Chromium Complexes .

Lentz Dieter: Tetrakis(trifluoromethyl isocyanide)nickel, $\mathrm{Ni}\left(\mathrm{CNCF}_{3}\right)_{4}$, and $\mathrm{Bis}[\mu$-(trifluoromethyl isocyanide)-( $\eta$-cyclopentadienyl)nickel], $\left[\mathrm{Ni}\left(\mu-\mathrm{CNCF}_{3}\right)\left(\eta-\mathrm{C}_{5} \mathrm{H}_{5}\right)\right]_{2} \ldots$

Brink Klaus and Mattes Rainer: Mesylhydroxylamines, VI: Oxidation of $N$-Mesylhydroxylamines - Vibrational Spectra and Structure of 1,2-Dimesyl-1,2-dimethoxy-

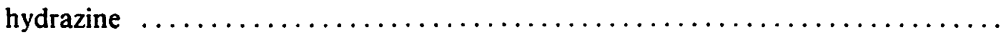

Lang Heinrich, Mohr Gerhard, Scheidsteger Olaf, and Huttner Gottfried: Halogen-Metal Exchange in Coordinated Halophosphanes: Synthesis of "Phosphinidene" Complexes

Boese Roland, Köster Roland, and Yalpani Mohamed: The Colour of Chelates of Boron. An $X$-Ray Structural Investigation of Bis(4-methylphenyl)boryl and 9-Borabicyclo-

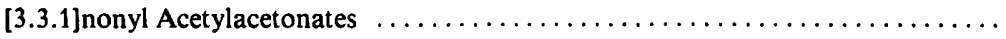

Wadepohl Hubert, Pritzkow Hans, and Siebert Walter: Synthesis and Structure of Platinum-Containing Triple-Decker and Tetra-Decker Complexes ............

Appel Rolf, Knoch Falk, and Zimmermann Rolf: Low Coordinated Phosphorus Compounds, 29: Diels-Alder Reactions of 2,3-Dimethylbutadiene with Stable Phospha-

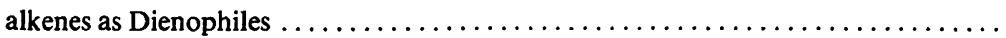

\section{ORGANIC CHEMISTRY}

Lösel Walter and Daniel Helmut: 3,4-Dihydroisoquinolines, I: 5,6-Dihydropyrrolo$[2,1-a]$ isoquinolines via Rearrangement of 1-(3-Furyl)-3,4-dihydroisoquinolines ....

Diercks Rainer and Dieck tom Heindirk: Diazadienes as Controlling Ligands in Homogeneous Catalysis, IX: Catalytic Cyclotetramerization of Propynoic Esters ........

Wamhoff Heinrich, Wald Klemens, Kirfel Armin, Farkas Lajos, Samimi Nabi, and Will Georg: Reactions of Uracils, 5: Bridged 1,2,5,6-Tetrazocanes with Uracil and Urazole Bridges by Dimerization of 5-(1,2,4-Triazolidin-1-yl)uracils .............

Zarkadis Antonios K., Neumann Wilhelm P., Marx Rainer, and Uzick Wolfram: Sterically Hindered Free Radicals, XII: The Radical tert-Butyldiphenylmethyl, Its Dimer, and Their Autoxidation. A Reinvestigation 
Kaupp Gerd and Knichala Bernd: Quantitative [1,3,2,3]-Elimination of Water from

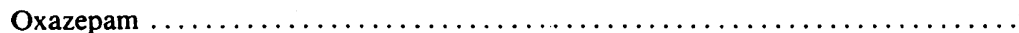

Effenberger Franz and Brodt Werner: 2(1H)-Pyridone as Leaving Group in Acylation Reactions - Applications in Peptide Synthesis $\ldots \ldots \ldots \ldots \ldots \ldots \ldots \ldots \ldots \ldots$

Weiske Thomas, Halim Hermann, and Schwarz Helmut: C-C Cleavage of Ionized Carboxylic Acids as Reversal of [1,4] Additions of Alkyl Radicals to Protonated $\alpha, \beta$ Unsaturated Acids. - On the Role of Enol Cation Radicals as Reactive Intermediates in Isomerization/Dissociation Reactions in the Gas Phase ...............

Döpp Dietrich, Krüger Carl, Makedakis George, and Nour-el-Din Ahmed Moukhtar: Indolenine Oxides, IX: Novel Polycyclic Linearly Conjugated Cyclohexadiene Imines from Rearrangement of Unstable Tetrahydroisoxazolo $[2,3-a]$ indoles $\ldots \ldots \ldots \ldots$

Quast Helmut and Nahr Uwe: Photoextrusion of Nitrogen from 1,4-Dihydro-1-phenyl$5 \mathrm{H}$-tetrazol-5-ones and -thiones. Benzimidazolones and Carbodiimides ..........

Sohár Pál, Lázár János, and Bernáth Gábor: Isolation and Structure Elucidation of the By-Product Formed in the Aminomethylation of $\alpha$-Methylstyrene . . . . . . . . . .

Siegel Herbert, Eisenhuth Ludwig, and Hopf Henning: Alkynes and Cumulenes, XVII: Photoaddition of Vinylacetylene to other Unsaturated Hydrocarbons ...........

Anke Lutz and Weyerstahl Peter: Inter- versus Intramolecular Friedel-Crafts Reaction of

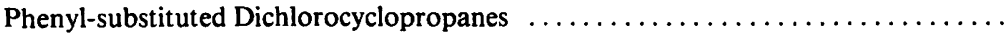

Daub Jörg, Lüdemann Hans-Dietrich, Michna Martin, and Strobl Reinhard M.: On the Correspondence of Heteroatom-stabilized Carbocations and the CycloheptatrieneNorcaradiene Equilibrium

Hoffmann Reinhard W. and Barth Wolfgang: Carbene Reactions, XVII: Nitrile Ylides via 1,1-Elimination

Kupfer Rainer, Würthwein Ernst-Ulrich, Nagel Michael, and Allmann Rudolf: 1-Alkoxy2-azaallenium Salts: $X$-Ray Crystallographic and Quantum Mechanical Structure Studies

Verkoyen Carl and Rademacher Paul: Reduction of $\beta$-Lactams, III: Synthesis of $\beta$-Thiolactams and Reduction with Raney Nickel $\ldots \ldots \ldots \ldots \ldots \ldots \ldots \ldots \ldots \ldots$

Yalpani Mohamed and Wilke Günther: Hydrated Oxocarbons, IV: Silylation of 1,1-Di-

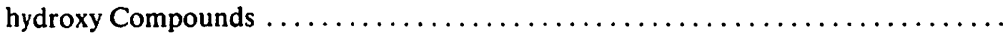

Boese Roland, Köster Roland, and Yalpani Mohamed: The Colour of Chelates of Boron. An $X$-Ray Structural Investigation of Bis(4-methylphenyl)boryl and 9-Borabicyclo[3.3.1]nonyl Acetylacetonates

Horner Leopold and Lindel Hans: Organophosphorus Compounds, 111: Phosphinic and Thiophosphinic Cyanides as Fluorescent SH-selective Reagents $\ldots \ldots \ldots \ldots \ldots \ldots$

Bäuml Englbert and Mayr Herbert: Silver Trifluoroacetate Initiated Reactions of Chlorotriphenylallene with Cyclopentadiene - Vinyl Cation Type Cycloadditions of Alle-

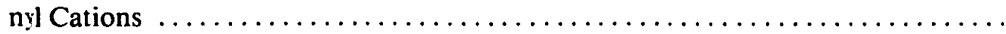

Bäumi Englbert and Mayr Herbert: Cycloadditions of the Triphenylallenyl Cation with Cyclopentadiene - Investigation of the Reaction Mechanism under Stable Ion Con-

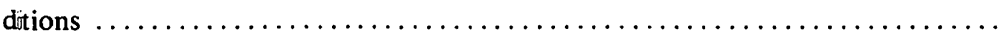

Maier Günther, Roth Cornelia, and Schmitt Reinhart K.: Stereoselectivity in the Hydride Reduction of Acyclic Diketones (1,2-, 1,3-, 1,4-, and 1,5-Induction) ............ 
Maier Günther, Schmitt Reinhart K., and Seipp Ulrich: Reversal of the Diastereoselectiv-

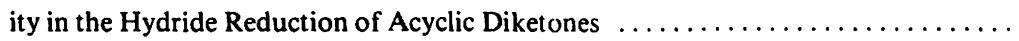

Effenberger Franz, Ziegler Thomas, and Schönwälder Karl-Heinz: Enol Ethers, XVI: Synthesis of 4-Hydroxy-2 $H$-pyran-2-ones

Lenoir Dieter and Frank Robert M.: Polar Effects of 4-Substituents in the Solvolysis of Tetracyclo[6.2.1.1 $1^{3,6} \cdot 0^{2,7}$ ]dodecan-11-yl Triflates. Through-Space vs. Inductive

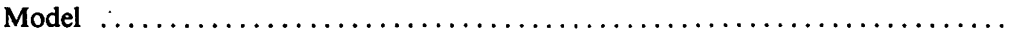

Bauer Walter, Laube Thomas, and Seebach Dieter: Crystal and Molecular Structure of a THF-solvated Lithium Amide Enolate Dimer . .................... 764

Gotthardt Hans, Feist $\nmid$ Ulrich, and Schoy-Tribbensee Sabine: Synthesis and Properties of

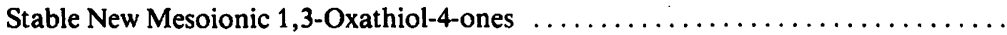

Gotthardt Hans and Feist $\dagger$ Ulrich: A New Entry to Alkylthio(trifluoroacetyl)furans by Way of Thermal [3 + 2]Cycloaddition Reactions of New Mesoionic 1,3-Oxathiol-4-

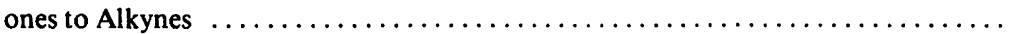

Staab Heinz A., Jörns Manfred, Krieger Claus, and Rentzea Marina: Electron DonorAcceptor Compounds, XXXVI: Quinones and Quinhydrones of the [2.2]- and [3.3]-

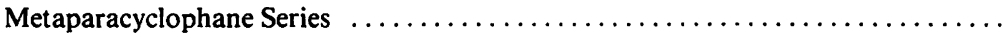

Eilbracht Peter, Balß Erika, and Acker Michael: Regio- and Stereoselectivity of the Hydrocarbonylating Cyclization of 1,4-Dienes with Carbon Monoxide: Synthesis of

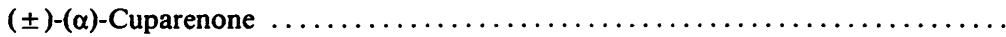

Hashem Md. Abul, Hülskämper Ludwig, and Weyerstahl Peter: Thermolysis and Photolysis of Hexachloro-tris- $\sigma$-homotropone and Related Compounds .............

Griesbaum Karl and Meister Martin: Disproval of Claimed Unusually Stable Primary Ozonides of 1,4-Dichloro-2-butenes 
Acker, M. s. Eilbracht, $P$. 825

Allmann, R. s. Kupfer, R. 643

Ambach, E. s. Beck, $W$. 444

Anke, L. und Weyerstahl, $P$. 613

Appel, R., Knoch, F. und

Zimmermann, $R$. 814

Bäuml, E. und Mayr, $H$. 683,694

Balß, E. s. Eilbracht, $P$. 825

Barth, W. s. Hoffmann, R. W. 634

Bauer, W., Laube, T. und Seebach, D. . . 764

Beck, W., Ambach, E. und Nagel, U. . . 444

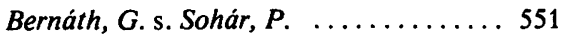

Boese, R., Köster, R. und Yalpani, M. . 670

Brink, K. und Mattes, $R$. . . . . . . . 564

Brodt, W. s. Effenberger, F. . ..... 468

Daniel, $H$. s. Lösel, $W$. 413

Daub, J., Lüdemann, H.-D.,

Michna, M. und Strobl, R. M. . . 620

Dieck, tom, H. s. Diercks, $R$. . . . . 428

Diercks, $R$. und Dieck, tom, H. ..... 428

Döpp, D., Krüger, C., Makedakis, G.

und Nour-el-Din, A. M. . . . . . 510

Effenberger, $F$. und Brodt, $W . \ldots \ldots .468$

-, Ziegler, T. und Schönwälder, K.-H. . 741

Eilbracht, P., Balß, E. und

Acker, M. ............. 825

Eisenhuth, L. s. Siegel, H. . . . . . . . 597

Farkas, L. s. Wamhoff, H. . . . . . 436

Feist $\dagger, U$. s. Gotthardt, H. . . . 774, 785

Frank, R. M. s. Lenoir, D. . . . . . . 753

Gotthardt, $H$. und Feist,$+ U . \ldots \ldots 785$

-, Feist †, U. und Schoy-Tribbensee, S. . 774

Griesbaum, K. und Meister, M. . . . . 845

Halim, H. s. Weiske, T. ....... 495

Hashem, M. A., Hülskämper, L. und

Weyerstahl, $P$. .......... 840

Hoffmann, R. W. und Barth, W. . . . 634

Hopf, H. s. Siegel, H. ......... 597

Horner, L. und Lindel, H. . . . . . . 676

Hülskämper, L. s. Hashem, M. A. . . . . 840
Huppmann, P. und Seppelt, K. . . . . 457

Huttner, G. s. Lang, H. . . . . . . . . 574

Jörns, M. s. Staab, H. A. . . . . . . 796

Kaupp, G. und Knichala, B. ....... 462

Kirfel, A. s. Wamhoff, H. ........ 436

Knichala, B. s. Kaupp, G. . . . . . . 462

Knoch, F. s. Appel, R. . . . . . . . 814

Köster, R. s. Boese, R. . . . . . . . 670

Krieger, C. s. Staab, H. A. ........ 796

Krüger, C. s. Döpp, D. ......... 510

Kumpfmüller, F., Nölle, D., Nöth, $H$. ,

Pommerening, $H$. und Staudigl, $R$. . 483

Kupfer, R., Würthwein, E.-U.,

Nagel, M. und Allmann, R. . . . . 643

Lang, H., Mohr, G., Scheidsteger, $O$. und Huttner, $G . \ldots \ldots \ldots \ldots \ldots 574$

Laube, T. s. Bauer, W. ......... 764

Lázár, J. s. Sohár, P. . .......... 551

Lenoir, D. und Frank, R. M. . . . . 753

Lentz, D. . . . . . . . . . . . . . . 560

Lindel, H. s. Horner, L. . . . . . . . 676

Lösel, $W$. und Daniel, $H . \ldots \ldots \ldots \ldots 413$

Lüdemann, H. D. s. Daub, J. . . . . . . 620

Maier, G., Roth, C. und Schmitt, R. K. . 704

-, Schmitt, R. K. und Seipp, U. ...... 722

Makedakis, G. s. Döpp, D. . ...... 510

Marx, R. s. Zarkadis, A. K. . ...... 450

Mattes, R. s. Brink, K. . . . . . . . 564

Mayr, H. s. Bäuml, E. . . . . . . . 683, 694

Meister, M. s. Griesbaum, K. ...... 845

Michna, M. s. Daub, J. . . . . . . . 620

Mohr, G. s. Lang, H. . . . . . . . . . 574

Nagel, M. s. Kupfer, R. . . . . . . . 643

Nagel, U. s. Beck, W. . . . . . . . . . 444

Nahr, U. s. Quast, H. ............ 526

Neumann, W. P. s. Zarkadis, A. K. . . 450

Nölle, D. s. Kumpfmüller, $F$. . . . . 483

Nöth, H. s. Kumpfmüller, F. . . . . 483

Nour-el-Din, A. M. s. Döpp, D. . . . . 510

Pommerening, H. s. Kumpfmüller, F. . . 483 
Pritzkow, H. s. Wadepohl, H. ...... 729

Quast, $H$. und Nahr, U. .......... 526

Rademacher, $P$. s. Verkoyen, $C$. . . . 653

Rentzea, M. s. Staab, H. A. ....... 796

Roth, C. s. Maier, G. . . . . . . . .. 704

Samimi, N. s. Wamhoff, $H . \ldots \ldots \ldots 436$

Scheidsteger, O. s. Lang, $H . \ldots \ldots \ldots 574$

Schmitt, R. K. s. Maier, G. ...... 704, 722

Schönwälder, K.-H. s. Effenberger, $F$. . 741

Schoy-Tribbensee, S. s. Gotthardt, H. . . 774

Schwarz, H. s. Weiske, T. ........ 495

Seebach, D. s. Bauer, W. . . . . . . . . 764

Seipp, U. s. Maier, G. . ......... 722

Seppelt, K. s. Huppmann, P. ....... 457

Siebert, W. s. Wadepohl, H. . . . . ... 729

Siegel, H., Eisenhuth, L. und Hopf, H. . 597

Sohár, P., Lázár, J. und Bernáth, G. . .. 551

Staab, H. A., Jörns, M., Krieger, C. und Rentzea, $M$. 796

Staudigl, R. s. Kumpfmüller, $F$. ..... 483

Strobl, R. M. s. Daub, J. . . . . . . . . 6 $6 \hat{z 0}$
Uzick, W. s. Zarkadis, A. K. ....... 450

Verkoyen, $C$. und Rademacher, P. .... 653

Wadepohl, $H$., Pritzkow, $H$. und

Siebert, $W$. 729

Wald, $K$. s. Wamhoff, $H$. 436

Wamhoff, H., Wald, K., Kirfel, A.,

Farkas, L., Samimi, N. und Will, G. 436 Weber, L. und Wewers, $D$. ........ 541

Weiske, T., Halim, H. und Schwarz, H. . 495 Wewers, D. s. Weber, L. .......... 541

Weyerstahl, P. s. Anke, L. ........ 613

- s. Hashem, M. A. ............ 840

Wilke, G. s. Yalpani, M. ......... 661

Will, G. s. Wamhoff, H. ........ 436

Würthwein, E.-U. s. Kupfer, R. . . . . 643

Yalpani, M. und Wilke, G. ........ 661

- s. Boese, R. ................. 670

Zarkadis, A. K., Neumann, W. P., Marx, $R$. und Uzick, $W . \ldots \ldots \ldots 450$

Ziegler, $T$. s. Effenberger, $F$. . . . . 741

Zimmermann, R. s. Appe!, R. . ..... 814 


\title{
Cycloadditionen des Triphenylallenyl-Kations mit Cyclopentadien - Studium des Reaktionsmechanismus unter stabilen Ionen-Bedingungen
}

\author{
Englbert Bäuml*) und Herbert Mayr*,*) \\ Institut für Organische Chemie der Universität Erlangen-Nürnberg, \\ Henkestr. 42, D-8520 Erlangen
}

Eingegangen am 6. Februar 1984

Das Triphenylallenyl-Kation (5) geht mit Cyclopentadien eine konzertierte [4 +2$]$ - sowie eine stufenweise $[2+2]$-Cycloaddition ein. Die Reaktionsmechanismen werden durch NMR-spektroskopische Beobachtung der intermediären Carbenium-Ionen aufgeklärt.

\section{Cycloadditions of the Triphenylallenyl Cation with Cyclopentadiene - Investigation of the Reaction Mechanism under Stable Ion Conditions}

The triphenylallenyl cation (5) reacts with cyclopentadiene in a concerted [4 +2$]$ and a stepwise $[2+2]$ cycloaddition. The reaction mechanisms are elucidated by NMR spectroscopic observation of the intermediate carbenium ions.

In der vorstehenden Arbeit ${ }^{1)}$ berichteten wir, daß Chlortriphenylallen (1) mit Cyclopentadien und Silbertrifluoracetat zu Allenylcyclopentenyl- sowie zu [3.2.0]- und [2.2.1]-bicyclischen Allyl-Derivaten reagiert (Gl. 1).

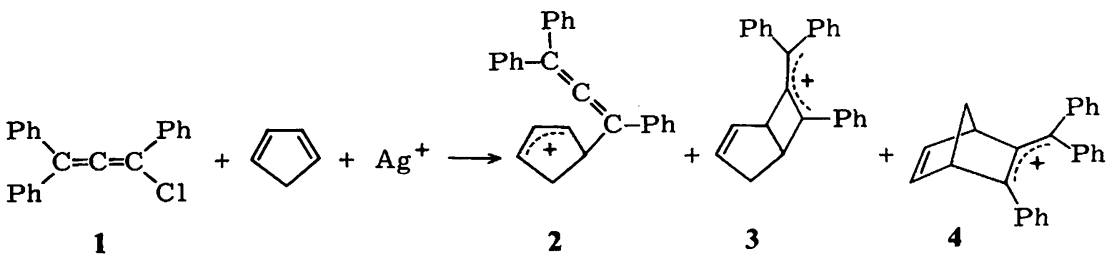

Wegen der zweifachen Phenylsubstitution am $\mathrm{sp}^{2}$-Kohlenstoff des intermediären Triphenylallenyl-Kations (5) wird der nucleophile Angriff an das sp-Ende abgedrängt, so daß 5 im Gegensatz zu den früher studierten Allenylkationen ${ }^{2)}$ ein Vinylkation-analoges Reaktionsverhalten ${ }^{3)}$ zeigt. Um Einblick in die Reaktionsmechanismen zu gewinnen, haben wir die Additionen von 5 unter stabilen Ionen-Bedingungen studiert. Dies eröffnet die Möglichkeit, einige der postulierten intermediären Carbenium-Ionen direkt spektroskopisch zu beobachten und ihre wechselseitigen Umlagerungen zu verfolgen.

*) Neue Anschrift: Institut für Chemie, Medizinische Hochschule Lübeck, Ratzeburger Allee 160, D-2400 Lübeck 1.

(C) VCH Verlagsgesellschaft mbH, D-6940 Weinheim, 1985

0009-2940/85/0202-0694\$02.50/0 


\section{Reaktionen des Triphenylallenyl-hexachloroantimonats mit Cyclopentadien}

a) Darstellung von Triphenylallenyl-hexachloroantimonat

Beim Versuch, 5 aus 1 und Antimonpentachlorid in situ zu erzeugen und an Cyclopentadien zu addieren, entstanden komplexe Produktgemische, so daß es günstig erschien, das Salz 5-SbCl- in Substanz darzustellen. Wegen der hohen Reaktivität des Allenylkations 5 gegenüber dem Allen 1 ist es dabei notwendig, den Kontakt zwischen dissoziiertem (5) und undissoziiertem Material (1) möglichst kurz zu halten. Dies gelingt, wenn eine Lösung von 1 in Pentan tropfenweise zu einer Lösung von Antimonpentachlorid gegeben wird, weil sich das hierbei entstehende Salz $\left(5-\mathrm{SbCl}_{6}^{-}\right)$spontan in Form dunkelroter Kristalle aus dem unpolaren Solvens ausscheidet.

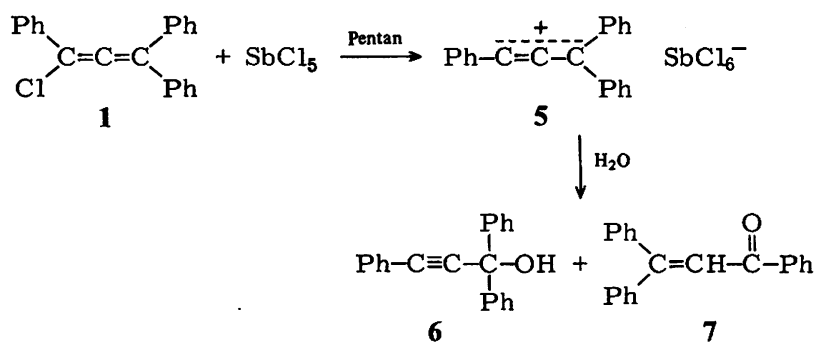

Im IR-Spektrum (Abb. 1) zeigt 5-SbCl- eine Kumulenbande bei $2145 \mathrm{~cm}^{-1}$, die zwischen der Allen-Absorption von $1\left(1958 \mathrm{~cm}^{-1}\right)^{4)}$ und der Alkinbande des Triphenylpropinols (6) $\left(2220 \mathrm{~cm}^{-1}\right)^{4)}$ liegt. Im fernen IR-Bereich findet sich eine Bande bei $350 \mathrm{~cm}^{-1}$, die der antisymmetrischen Valenzschwingung des HexachloroantimonatAnions $^{5)}$ zugeordnet werden kann.

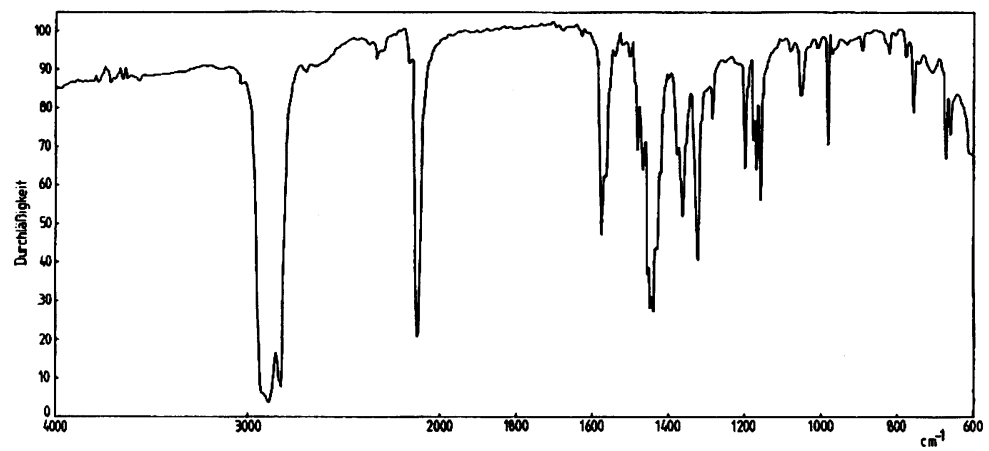

Abb. 1. IR-Spektrum von Triphenylallenyl-hexachloroantimonat (5-SbCl- ) in Nujol

Bei der Hydrolyse mit wäßriger Kaliumcarbonatlösung entstehen 6 und 7 im Verhältnis $44: 56$.

Chem. Ber. 118 (1985) 


\section{b) Cyclopentadien und 5-SbCl-}

Versetzt man eine Lösung von $5-\mathrm{SbCl}_{6}^{-}$in $\mathrm{SO}_{2}$ bei $-30^{\circ} \mathrm{C}$ mit einer Lösung von Cyclopentadien in Dichlormethan, so erhält man nach alkalischer Hydrolyse mit $29 \%$ Gesamtausbeute die bereits bekannten [3.2.0]-Bicyclen $8 \mathbf{a}$ und $\mathbf{8 b}^{1)}$ sowie einen sekundären Alkohol, dem vermutlich die Struktur 9 zukommt. Das komplexe ${ }^{1} \mathrm{H}$-NMRSpektrum von 9 läßt sich durch Zusatz von $\mathrm{Eu}(\mathrm{fod})_{3}$ auflösen. Dabei zeigt sich, daß das zur Hydroxygruppe geminale Proton mit dem benachbarten Brückenkopfproton nicht koppelt, woraus die exo-Stellung der OH-Gruppe resultiert. Der Vergleich der beobachteten und nach der $M c$ Connell-Gleichung ${ }^{6}$ berechneten induzierten Verschiebungen läßt keine Aussage über die Stereochemie an C-4a zu. Eine Struktur, bei der sich die OH-Gruppe an C-3 befindet, läßt sich spektroskopisch nicht ausschließen, ist aber aus mechanistischen Gründen weniger wahrscheinlich.

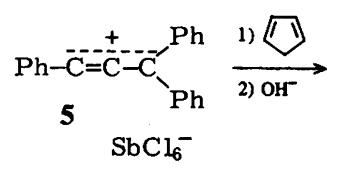

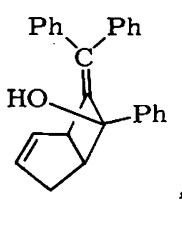

$8 \mathbf{a}$

39

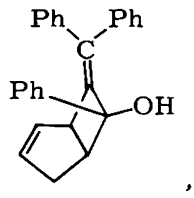

8b

50

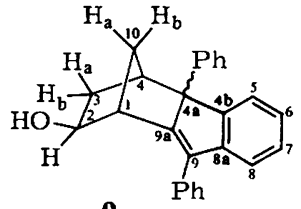

9

11

\section{Reaktion des Triphenylpropinols (6) mit Cyclopentadien in Gegenwart von Fluorsulfonsäure}

Anders als bei der Verwendung von Antimonpentachlorid erwies es sich hier als günstiger, das Triphenylallenyl-Kation in situ zu erzeugen. Tropft man 6 und Cyclopentadien bei -45 bis $-50^{\circ} \mathrm{C}$ gemeinsam zu Fluorsulfonsäure in flüssigem Schwefeldioxid, so isoliert man nach alkalischer Aufarbeitung mit 36\% Gesamtausbeute die diastereomeren Alkohole 8a und 8 b sowie die Carbonylverbindungen 7 und 10.
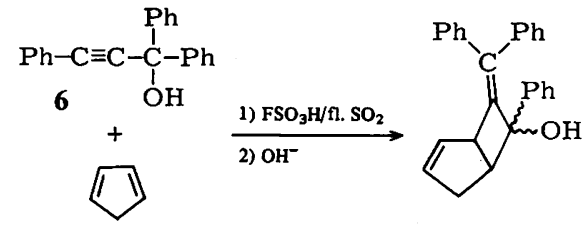

8a: $43 \%$

8b: $19 \%$

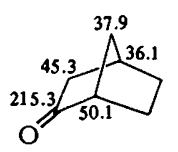

(zum ${ }^{13} \mathrm{C}-\mathrm{NMR}$ Vergleich mit 10)

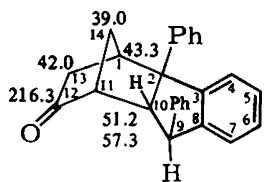<smiles>O=C(C=C=Cc1ccccc1)c1ccccc1</smiles>

$10: 16 \%$

$7: 22 \%$

Verbindung $10 \mathrm{mu}$ auf Grund der Infrarot-Absorption bei $1740 \mathrm{~cm}^{-1}$ ein Keton sein. Der Vergleich mit dem ${ }^{13} \mathrm{C}$-NMR-Spektrum von 2-Norbornanon deutet auf das Vorliegen eines entsprechenden Fragments hin. 
Da nach ${ }^{13} \mathrm{C}$-NMR keine nichtaromatischen $\mathrm{sp}^{2}$-Kohlenstoffe vorliegen, ergibt sich aus der Zahl der Doppelbindungsäquivalente, daß 10, wie 9, einen zusätzlichen Ring enthält. Die unten beschriebene Bildung von 10 aus 11 und 13 legt die angegebene Konstitutionsformel nahe.

Seine Konfiguratior ergibt sich aus den H,H-Kopplungskonstanten. So zeigt die 5.6-Hz-Kopplung zwischen 11-H und 10-H die exo-Stellung von 10-H, d.h., daß der Indan-Teil an den Norbornan-Teil endo-anelliert ist. Aus der 3.2-Hz-Kopplung zwischen 9-H und 10-H folgt deren trans-Stellung, wodurch die Stellung der 9-Phenylgruppe festgelegt ist.

\section{Bildungsweise der Tricyclen 9 und 10}

Es ist naheliegend, 9 und 10 als Folgeprodukte des Allylkations 4 anzusehen. Um dies zu beweisen, wurden die Alkohole 11-13 mit überschüssiger Fluorsulfonsäure in flüssigem Schwefeldioxid behandelt. In allen Fällen erhielt man bei $-60^{\circ} \mathrm{C}$ ein Gemisch zweier Carbenium-Ionen, dessen Zusammensetzung sich beim Erwärmen auf $-30^{\circ} \mathrm{C}$ nicht veränderte. Tropft man $11-13$ dagegen bei $-78^{\circ} \mathrm{C}$ zu Fluorsulfonsäure in $\mathrm{CDCl}_{3}$ und läßt dann auf Raumtemperatur aufwärmen, so beobachtet man lediglich eines der beiden bei $-60^{\circ} \mathrm{C}$ vorliegenden Ionen, dem sich die Struktur 17 zuordnen läßt.

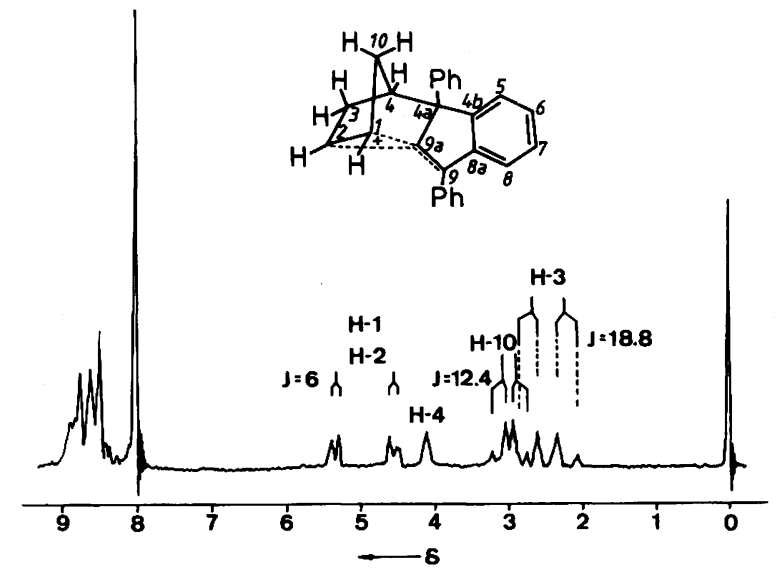

Abb. 2. ${ }^{1} \mathrm{H}$-NMR-Spektrum des Kations 17 (60 MHz, TMS extern) in $\mathrm{CDCl}_{3}$

Das ${ }^{1} \mathrm{H}$-NMR-Spektrum von 17 (Abb. 2) zeigt, daß die positive Ladung über zwei Arylreste delokalisiert ist, während der dritte Benzolkern praktisch ungeladen ist (5-Protonen-Singulett bei $\delta=7.97$ ). Im ${ }^{13} \mathrm{C}$-NMR-Spektrum findet man ein Singulett bei $\delta=201.1$, woraus hervorgeht, daß sich ein Großteil der positiven Ladung auf C-9 befindet. Neben zwei aromatischen Ringen tragen auch C-1 und C-2 einen Teil der positiven Ladung. Nimmt man das ${ }^{13} \mathrm{C}$-NMR-Spektrum von 17 bei 0 und $-50^{\circ} \mathrm{C}$ auf, so beobachtet man die gleichen chemischen Verschiebungen. Daraus geht hervor, daß 17 eine statische Spezies darstellt, daß also nicht ein Satz miteinander im Gleichgewicht befindlicher Carbenium-Ionen $\left(\mathrm{C}^{+}\right.$an Position 1, 2 oder 9) vorliegt. Wie Tab. 1 zeigt, 
sind die für 17 beobachteten ${ }^{13} \mathrm{C}$-NMR-chemischen Verschiebungen in Einklang mit den von Sorensen für symmetrische Nortricyclylcarbinyl-Kationen beschriebenen Werten? .

Tab. 1. ${ }^{13}$ C-NMR-Daten einiger Nortricyclylcarbinyl-Kationen

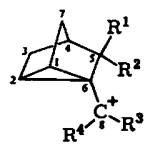

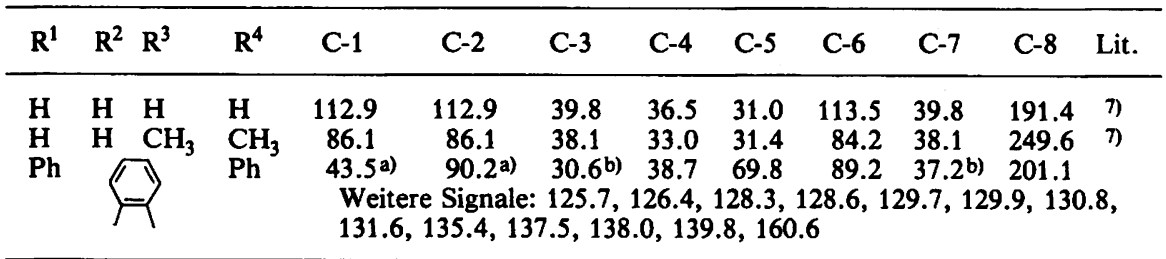

a) b) Zuordnung nicht eindeutig möglich.

Schema 1<smiles>OC(C1=C(c2ccccc2)C2C=CC1C2)(c1ccccc1)c1ccccc1</smiles>

11<smiles>OC1(c2ccccc2)C(=C(c2ccccc2)c2ccccc2)C2C=CC1C2</smiles>

12<smiles>OC1C2CCC(c3ccccc3)(C2)C1C(c1ccccc1)c1ccccc1</smiles>

13<smiles>CCC(C)C(C)C</smiles><smiles>C1=CC2CC1[C+](c1ccccc1)C(c1ccccc1)C2c1ccccc1</smiles>

4<smiles>C1=CC2CC1C1=C(c3ccccc3)c3ccccc3C12[R]1Cc2ccccc21</smiles>

14<smiles>PC1=CC=CC2(c3ccccc31)C1C=CC(C1)C2c1ccccc1</smiles>

15

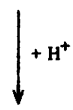

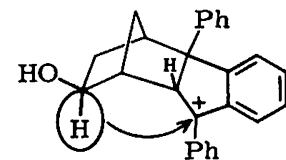

16

1,4-H Verschiebung<smiles></smiles>

18

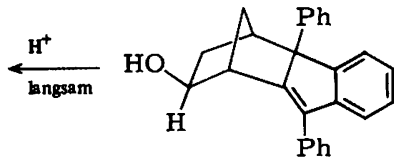

9 (4a-Ph: exo)

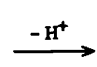

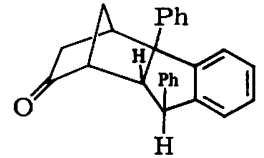

10
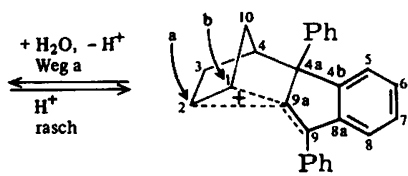

17

$\mathrm{H}^{+} \Uparrow \underset{\text { Weg b }}{+\mathrm{H}_{2} \mathrm{O}_{1}-\mathrm{H}^{+}}$

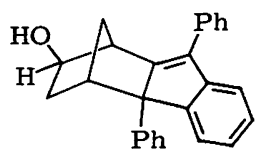

9 (4a-Ph: endo)

Chem. Ber. 118 (1985) 
Schema 1 zeigt, daß das Allylkation 4, das beim Behandeln von 11 - 13 mit Fluorsulfonsäure entsteht, zunächst den für Phenylallyl-Kationen typischen Fünfringschluß eingeht ${ }^{8)}$. Dabei entsteht das Kation 14, das sich durch Deprotonierung und Reprotonierung über 15 zu 17 umlagern kann. Alternativ zu diesem in Schema 1 gezeigten Weg könnte 14 auch durch Protonierung an C-3 in ein Dikation übergehen, dessen Deprotonierung ebenfalls das spektroskopisch beobachtete Carbokation 17 ergäbe. Je nachdem, ob der nucleophile Angriff von Wasser an C-1 oder C-2 erfolgt, kann es zur Bildung zweier diastereomerer Alkohole (9) kommen, die sich in der Konfiguration an C-4a unterscheiden. Wie oben erwähnt, läßt sich die Stereochemie des von uns erhaltenen Isomeren spektroskopisch nicht ermitteln.

9 liefert wie 11-13 beim Einbringen in überschüssige Fluorsulfonsäure das Kation 17. Behandelt man 11 oder 13 dagegen mit äquimolaren Mengen Fluorsulfonsäure, so kommt es zur Umlagerung in 10; entsprechende Reaktionen sind für die Alkohole 9 und $12 \mathrm{zu}$ erwarten. Dieses Verhalten wird in der unteren Hälfte von Schema 1 gedeutet.

Arbeitet man mit überschüssiger Fluorsulfonsäure, so liegt das aus den Alkoholen abgespaltene Wasser in protonierter Form vor, steht also nicht mehr als Nucleophil zur Verfügung. Werden dagegen nur äquimolare Mengen an Fluorsulfonsäure verwendet, so können durch Wasseranlagerung an 17 die Alkohole 9 gebildet werden. Das Isomere 9 mit dem endo-anellierten Indenring (4a-Ph:exo) kann nun durch Protonierung an C-9a das Kation 16 ergeben, das durch 1,4-Wasserstoffverschiebung in 18, die protonierte Form des Ketons 10 übergeht. Dieser Mechanismus erklärt die aus den HH-Kopplungskonstanten abgeleitete Stereochemie von 10. Somit ist 9 das kinetisch, $\mathbf{1 0}$ das thermodynamisch gelenkte Folgeprodukt von 17.

\section{Mechanismus der Triphenylallenyl-Kation-Cycloadditionen: Konzertiert oder stufenweise?}

Da nunmehr gesichert ist, daß die Tetracyclen 9 und 10 Folgeprodukte des [2.2.1]-Bicyclus 4 darstellen, ist es trotz der mäßigen in Abschnitt 1 und 2 beschriebenen Ausbeuten an Additionsprodukten eindeutig, daß zwischen dem Triphenylallenyl-Kation (5) und Cyclopentadien auch unter stabilen Ionen-Bedingungen die $[2+4]$-Cycloaddition

Schema 2

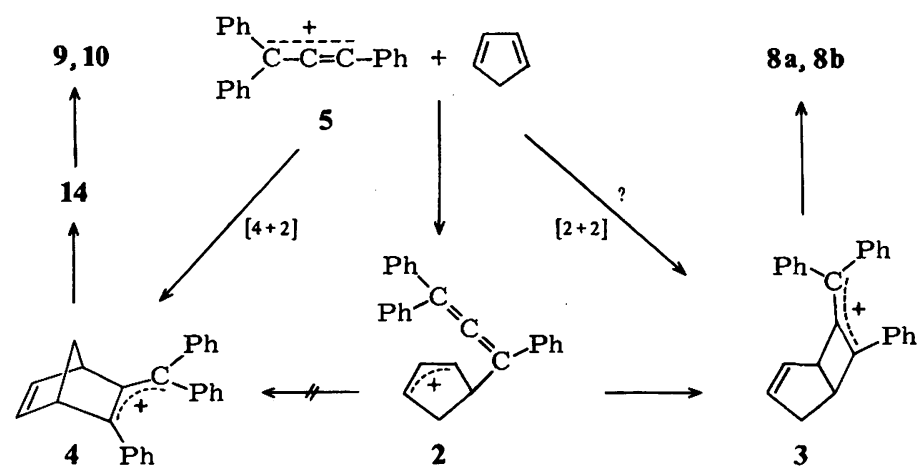

Chem. Ber. 118(1985) 
und die $[2+2]$-Cycloaddition nebeneinander ablaufen. Bei den Silbertrifluoracetatinitiierten Reaktionen in Pentan stellen Allenylcyclopentenole, wie 19, den Hauptanteil der Additionsprodukte dar ${ }^{1,9)}$. Dagegen wurden in dieser Arbeit unter stabilen IonenBedingungen keine Abfangprodukte von 2 isoliert, so daß man annehmen muß, daß 2 zu 3 oder zu 4 cyclisiert (Schema 2).

Rührt man fein gepulvertes $19^{1)}$ bei $-78^{\circ} \mathrm{C}$ in eine Lösung von 1.15 Äquivalenten Fluorsulfonsäure in Schwefeldioxid ein, so erhält man 3, das sich NMR-spektroskopisch beobachten läßt; d.h., 2 cyclisiert im Rahmen der NMR-Nachweisgrenze ausschließlich zu 3. Bei höherem Säure-Überschuß kommt es zur Addition von Fluorsulfonsäure an die Doppelbindung des Fünfrings. Das gleiche NMR-Spektrum beobachtet man, wenn $8 a$ in eine Lösung von Fluorsulfonsäure eingebracht wird. Im Gegensatz zu 4 (Abschnitt 3) zeigt 3 bei $-70^{\circ} \mathrm{C}$ keine Tendenz zur Cyclisierung. Das intakte [3.2.0]bicyclische System in 3 läßt sich auch durch alkalische Hydrolyse beweisen, die zu den Alkoholen $8 \mathbf{a}$ und $8 \mathrm{~b}$ führt.<smiles>CCCC1C2CC=CC1C(=C(c1ccccc1)c1ccccc1)C2</smiles>

19

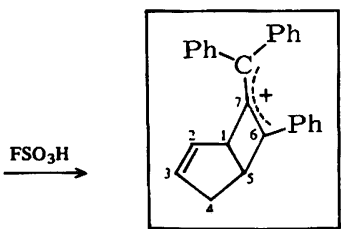

3

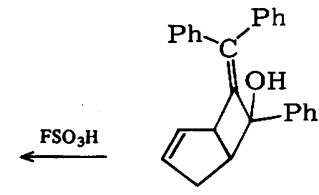

$8 \mathbf{a}$

Warum cyclisiert 2 zum Allylkation 3 und nicht zum weniger gespannten [2.2.1]-Bicyclus 4? Formel 20 zeigt, daß zwischen dem Allylkation-Fragment und dem AllenylTeil eine sterisch günstige 1,3-Wechselwirkung ( $m$ ) eintreten kann, die die Knüpfung der 1,4-Bindung einleitet. Dagegen ist für die Entstehung von 4 eine relativ große Winkeldeformation erforderlich, bevor eine Bindungsbeziehung zwischen den beiden $\pi$-Systemen auftreten kann.

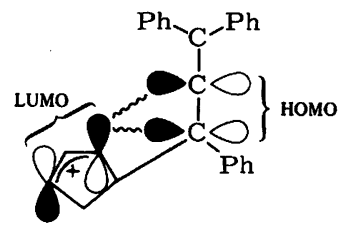

20

Da in Schema 2 die Cyclisierung $2 \rightarrow 4$ ausgeschlossen werden kann, muß 4 aus einer konzertierten [4 +2$]$-Cycloaddition hervorgehen. Die einzig verbleibende Unsicherheit in Schema 2 ist daher die Frage, ob der stufenweise $[2+2]$-Cycloadditionsmechanismus noch durch den konzertierten $\left[{ }_{\pi} 2_{\mathrm{s}}+{ }_{\pi} 2_{\mathrm{a}}\right]$-Prozeß begleitet ist. Da eine zwingende Notwendigkeit für die zusätzliche Annahme des konzertierten Prozesses nicht besteht, deuten wir diese Ergebnisse durch einen konzertierten [4 +2$]$ - und einen stufenweisen $[2+2]$-Mechanismus (Occams Prinzip ${ }^{10)}$ ). 


\section{Schlußfolgerungen}

Verzichtet man auf den Einsatz von „Occams razor“, so ergibt sich aus den Ergebnissen dieser und der vorstehenden Arbeit, daß die konzertierte $\left[\mathbf{2}_{\mathrm{s}}+{ }_{\pi} \mathbf{2}_{\mathrm{a}}\right]$-Cycloaddition des Triphenylallenyl-Kations (5) mit Cyclopentadien, sofern sie überhaupt stattfindet, dem stufenweisen Prozeß zumindest unterlegen ist. Wie bei den stufenweise cycloaddierenden Keteniminium-Ionen fehlt bei 5 ein energetisch hochliegendes HOMO, was neben dem dazu orthogonalen tiefliegenden LUMO Voraussetzung für eine konzertierte $\left[{ }_{\pi} 2_{\mathrm{s}}+{ }_{\pi} 2_{\mathrm{a}}\right]$-Cycloaddition sein soll ${ }^{11)}$. Es stellt sich daher die Frage, ob Vinylkationen, die orthogonal zum leeren p-Orbital ebenfalls kein hochliegendes HOMO besitzen, tatsächlich wie behauptet ${ }^{12)}$ die ${ }_{{ }_{\pi}}{ }_{\mathrm{a}} \mathrm{a}$-Komponenten par excellence für synchrone $\left[2_{\mathrm{s}}+{ }_{\pi} \boldsymbol{2}_{\mathrm{a}}\right]$-Cycloadditionen" darstellen oder ob sie diesen Rang an die Ketene abtreten müssen.

\section{Experimenteller Teil}

Allgemeine Vorbemerkungen und Geräte s. Lit. ${ }^{1)}$.

1. Synthese von Triphenylallenyl-hexachloroantimonat (5- $\left.\mathrm{SbCl}_{6}^{-}\right)$: $\mathrm{Zu}$ einer Lösung von $1.35 \mathrm{~g}$ (4.51 mmol) $\mathrm{SbCl}_{5}$ (unter $\mathrm{N}_{2}$-Schutz destilliert, Sdp. $69^{\circ} \mathrm{C} / 15$ Torr) in $25 \mathrm{ml} n$-Pentan wurde

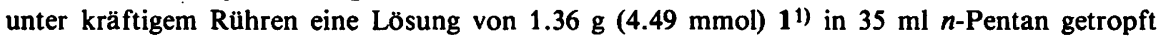
$\left(\mathrm{N}_{2}\right.$-Schutz). Es bildete sich ein roter Niederschlag. Nach dem Zutropfen wurde noch $15 \mathrm{~min}$ gerührt, dann der Niederschlag unter $\mathrm{N}_{2}$-Schutz mit einer Fritte abgesaugt, mit wenig Pentan nachgewaschen und im Ölpumpenvakuum getrocknet. Man erhielt $2.54 \mathrm{~g}(94 \%)$ rotes, an der Luft zersetzliches Pulver.

2. Umsetzung von 5-SbCl- mit Cyclopentadien: $1.94 \mathrm{~g}(3.22 \mathrm{mmol}) 5-\mathrm{SbCl}_{6}^{-}$wurden unter Stickstoffschutz in $70 \mathrm{ml}$ flüssigem $\mathrm{SO}_{2}$ bei $-30^{\circ} \mathrm{C}$ suspendiert. Dazu wurde innerhalb von $45 \mathrm{~min}$ unter Rühren eine Lösung von $0.32 \mathrm{~g}(4.8 \mathrm{mmol})$ Cyclopentadien in $10 \mathrm{ml}$ absol. Methylenchlorid getropft. Nach $6.5 \mathrm{~h}$ bei $-30^{\circ} \mathrm{C}$ wurde die Reaktionsmischung in eine auf $-5^{\circ} \mathrm{C}$ vorgekühlte Kaliumcarbonatlösung ( $60 \mathrm{~g} \mathrm{~K}_{2} \mathrm{CO}_{3}$ in $200 \mathrm{ml}$ dest. Wasser) gegossen und $200 \mathrm{ml}$ Methylenchlorid zugegeben. Als die Mischung Raumtemp. angenommen hatte, wurde die wäßrige Phase mit ca. $100 \mathrm{ml}$ konz. $\mathrm{K}_{2} \mathrm{CO}_{3}$-Lösung (bei Verwendung von $\mathrm{Na}_{2} \mathrm{CO}_{3}$-Lösung Aufarbeitung problematisch!) neutralisiert. Dabei erfolgte ein Farbumschlag der organischen Phase von Blaugrün nach Rot. Die organische Phase wurde abgetrennt, filtriert und über $\mathrm{CaCl}_{2}$ getrocknet. Nach dem Abziehen des Lösungsmittels blieben $1.08 \mathrm{~g}$ rotes Harz.

$4.86 \mathrm{~g}$ dieses Rohprodukts, die aus mehreren Ansätzen obiger Größenordnung gewonnen wurden, wurden durch Säulenchromatographie getrennt $(500 \mathrm{~g}$ Kieselgel, Laufmittelgradient: 11 Petrolether: Methylenchlorid = 1:1,61 Petrolether: Methylenchlorid = 1:2, 41 Methylenchlorid, 11 Ether). Nach $1.18 \mathrm{~g}$ eines nicht identifizierten komplexen Gemisches eluierte man $569 \mathrm{mg}$ (11.2\%) 8 $\mathrm{a}^{1}$ ), und weiteren $240 \mathrm{mg}$ einer unbekannten Substanz folgten $737 \mathrm{mg}(14.5 \%) 8 \mathrm{~b}^{1)}$ sowie eine Mischfraktion, aus der beim Zusatz von $\mathrm{CH}_{2} \mathrm{Cl}_{2} 160 \mathrm{mg}$ (3.2\%) 9 auskristallisierte.

(1 $\alpha, 2 \alpha, 4 \alpha)-2,3,4,4 a-T e t r a h y d r o-4 a, 9-d i p h e n y l-1,4-m e t h a n o-1 H$-fluoren-2-ol (9): Feine, farblose Nadeln vom Schmp. $168-170^{\circ} \mathrm{C}\left(\mathrm{CH}_{2} \mathrm{Cl}_{2}\right)$. - IR (KBr): $3400(\mathrm{OH}), 3040,2950(\mathrm{CH}), 1640$, $1630(\mathrm{C}=\mathrm{C}), 1490,1590$ (Aromatengerüst), 700,760,1055, 745, 785, 1440,1450 $\mathrm{cm}^{-1}$. ${ }^{1} \mathrm{H}-\mathrm{NMR}\left(\mathrm{CDCl}_{3}\right): \delta=1.17(\mathrm{mc} ; 2 \mathrm{H}), 1.52$ (br. s; $\left.1 \mathrm{H}, \mathrm{OH}\right), 1.99(\mathrm{mc} ; 2 \mathrm{H}), 3.25-3.62(\mathrm{~m} ; 3 \mathrm{H})$, $6.98-7.85(\mathrm{~m} ; 14 \mathrm{H}$, Aromaten-H).

Das ${ }^{1} \mathrm{H}$-NMR-Spektrum wurde durch Zusatz von Eu(fod) ${ }_{3}$ aufgelöst, und die beobachteten Verschiebungen wurden mit den berechneten verglichen (vgl. Lit. $\left.{ }^{1)}\right)$ :

Chem. Ber. $118(1985)$ 


\begin{tabular}{lcccc}
\hline & $\delta$ extrapol. & $\Delta_{\text {exp }}{ }^{\text {a) }}$ & $\Delta_{\text {ber }}{ }^{\text {b c) }}$ & $\Delta_{\text {ber }}{ }^{\text {b,d) }}$ \\
\hline $1-\mathrm{H}$ & 3.17 & 10.92 & 9.28 & 10.40 \\
$2-\mathrm{H}$ & 3.22 & 18.53 & 16.52 & 16.91 \\
$3 \mathrm{a}-\mathrm{H}$ & 1.12 & 12.13 & 11.08 & 11.34 \\
$3 \mathrm{~b}-\mathrm{H}$ & 0.96 & 6.28 & 6.59 & 7.38 \\
$4-\mathrm{H}$ & 3.28 & 3.71 & 5.06 & 4.40 \\
$10 \mathrm{a}-\mathrm{H}$ & 1.95 & 9.75 & 9.88 & 8.63 \\
$10 \mathrm{~b}-\mathrm{H}$ & 1.82 & 4.61 & 4.94 & 4.80 \\
\hline
\end{tabular}

a) $\Delta_{\exp }=\Delta \delta / \Delta(\mathrm{mol}$ Verschiebungsreagens $/ \mathrm{mol} \mathrm{Alkohol}) .-$ b) $K\left(3 \cos ^{2} \Theta-1\right) / r_{i}^{3}($ McConnell 6$)$; $\mathrm{O}-\mathrm{Eu}=3 \AA$; Winkel C-O-Eu $=130^{\circ}$; Interplanarwinkel 2-H, C-2, O, Eu $=0^{\circ}$. - c) 4a-Phenyl in exo-Position. - d) 4a-Phenyl in endo-Position.

Aus dem durch Eu(fod) ${ }_{3}$-Zusatz aufgelösten Spektrum lassen sich folgende Kopplungen entnehmen: $J_{1,2}<1 \mathrm{~Hz}, J_{3 \mathrm{a}, 4}=4.8 \mathrm{~Hz}, J_{3 \mathrm{a}, 3 \mathrm{~b}}=14.4 \mathrm{~Hz}, J_{3 \mathrm{~b}, 2}=6.4 \mathrm{~Hz}, J_{10 \mathrm{a}, 10 \mathrm{~b}}=10.4 \mathrm{~Hz}$.

${ }^{13} \mathrm{C}-\mathrm{NMR}\left(\mathrm{CDCl}_{3}\right): \delta=35.0,36.3(2 \mathrm{t}, \mathrm{C}-3, \mathrm{C}-10), 43.2(\mathrm{~d}, \mathrm{C}-4), 47.2(\mathrm{~d}, \mathrm{C}-1), 71.8(\mathrm{~s}, \mathrm{C}-4 \mathrm{a})$, 76.2 (d, C-2), 120.6, 124.6, 125.2, 126.3, 126.6, 127.8, 128.6 (Aromaten-C), 135.9, 142.9, 144.1, $146.5,150.3,156.4\left(6 \mathrm{~s}, 2 \mathrm{C}_{\mathrm{ipso}}, \mathrm{C}-4 \mathrm{~b}, \mathrm{C}-8 \mathrm{a}, \mathrm{C}-9, \mathrm{C}-9 \mathrm{a}\right) .-\mathrm{MS}(70 \mathrm{eV}): m / e=350\left(100 \%, \mathrm{M}^{+}\right)$, 332 (3), 322 (15), 306 (31), 281 (66), 280 (57), 165 (62).

$$
\mathrm{C}_{26} \mathrm{H}_{22} \mathrm{O}(350.5) \text { Ber. C } 89.11 \text { H } 6.33 \text { Gef. C } 88.36 \text { H } 6.16
$$

3. Umsetzung von Triphenylpropinol (6) mit Cyclopentadien in Gegenwart von Fluorsulfonsäure: $1.44 \mathrm{~g}$ (14.4 mmol) Fluorsulfonsäure wurden unter Stickstof fschutz in ca. $80 \mathrm{ml}$ flüssigem Schwefeldioxid gelöst und auf -45 bis $-50^{\circ} \mathrm{C}$ gekühlt. Dazu tropfte man eine Lösung von $3.93 \mathrm{~g}$ $(13.8 \mathrm{mmol}) 6$ und $1.21 \mathrm{~g}(18.3 \mathrm{mmol})$ Cyclopentadien in $10 \mathrm{ml}$ absol. Methylenchlorid innerhalb von $0.5 \mathrm{~h}$ zu. Nach $3.5 \mathrm{~h}$ wurde die dunkelrote Reaktionsmischung in $200 \mathrm{ml}$ auf $0^{\circ} \mathrm{C}$ vorgekühlte 2proz. $\mathrm{Na}_{2} \mathrm{CO}_{3}$-Lösung eingegossen. $200 \mathrm{ml}$ Methylenchlorid wurden zugesetzt und nach Aufwärmen auf Raumtemp. die wäßrige Phase mit konz. $\mathrm{Na}_{2} \mathrm{CO}_{3}$-Lösung neutralisiert. Die organische Phase wurde abgetrennt, zweimal mit je $50 \mathrm{ml}$ gesättigter $\mathrm{Na}_{2} \mathrm{CO}_{3}$-Lösung gewaschen und über $\mathrm{Na}_{2} \mathrm{SO}_{4}$ getrocknet. Nach dem Abziehen des Lösungsmittels blieben $5.22 \mathrm{~g}$ Rohprodukt. Dieses Rohprodukt wurde zur Entfernung von Polymeren über $20 \mathrm{~g}$ Kieselgel filtriert. Als Lösungsmittel diente Ether. Das so erhaltene Produktgemisch $(3.95 \mathrm{~g})$ wurde durch Säulenchromatographie getrennt $(500 \mathrm{~g}$ Kieselgel, 7 I Petrolether: Methylenchlorid $=1: 1,11$ Methylenchlorid, 11 Ether). Mit zunehmender Retentionszeit erhielt man $739 \mathrm{mg}$ (15.3\%) 891), $310 \mathrm{mg}$ (7.9\%) $7^{11)}, 324 \mathrm{mg}$ $(6.7 \%) 8 b^{1)}$ und $280 \mathrm{mg}(5.8 \%)$ 10. Die in den Zwischenfraktionen enthaltenen Produktgemische wurden nicht identifiziert.

exo-2,9-Diphenyltetracyclo[9.2.1. $0^{2,10} \cdot 0^{3,8}$ Jtetradeca-3,5,7-trien-12-on $\quad$ (10): $\quad$ Schmp. $154.5-155.5^{\circ} \mathrm{C} /$ Ether. - IR (KBr): 2980, 3020, 2940, $3060(\mathrm{CH}), 1740(\mathrm{C}=\mathrm{O}), 1490,1595$ (Aromatengerüst), 695, 745, 1145, $960 \mathrm{~cm}^{-1}$ (CH-wagging). - ' $\mathrm{H}-\mathrm{NMR}\left(\mathrm{CDCl}_{3}\right)$ bei $200 \mathrm{MHz}$ : $\delta=1.55\left(\mathrm{dd}, J_{13 \mathrm{a}, 13 \mathrm{~b}}=18.3 \mathrm{~Hz}, J_{1,13 \mathrm{a}}=4.6 \mathrm{~Hz} ; 1 \mathrm{H}, 13 \mathrm{a}-\mathrm{H}\right), 1.91-2.09(\mathrm{~m} ; 3 \mathrm{H}, 13 \mathrm{~b}-\mathrm{H}, 14 \mathrm{a}-\mathrm{H}$, $14 \mathrm{~b}-\mathrm{H}), 3.00\left(\mathrm{~d}, J_{10,11}=5.6 \mathrm{~Hz} ; 1 \mathrm{H}, 11-\mathrm{H}\right), 3.23\left(\mathrm{dd}, J_{10,11}=5.6 \mathrm{~Hz}, J_{9,10}=3.2 \mathrm{~Hz} ; 1 \mathrm{H}\right.$, $10-\mathrm{H}), 3.52\left(\mathrm{~d}, J_{1,13 \mathrm{a}}=4.6 \mathrm{~Hz} ; 1 \mathrm{H}, 1-\mathrm{H}\right), 4.38\left(\mathrm{~d}, J_{9,10}=3.2 \mathrm{~Hz} ; 1 \mathrm{H}, 9-\mathrm{H}\right), 6.97-7.35(\mathrm{~m}$; $14 \mathrm{H}$, Aromaten-Protonen). $-{ }^{13} \mathrm{C}-\mathrm{NMR}\left(\mathrm{CDCl}_{3}\right): \delta=39.0$ (t; C-14), 42.0 (t; C-13), 43.3 (d; C-1), 51.2, 57.3 (2 d; C-10, C-11), 61.2 (d; C-9), 64.2 (s; C-2), 125.0, 126.0, 126.3, 126.7, 127.8, 127.9, 128.3, 128.5 (Aromaten-C), 143.5, 145.7, 147.2, 147.9 (4 s; $2 \mathrm{C}_{\text {ipso }}, \mathrm{C}-3, \mathrm{C}-8$ ), 216.3 (s; $\mathrm{C}-12)$. - MS (70 eV): $m / e=350\left(34 \%, \mathrm{M}^{+}\right), 268(100), 267$ (23).

$$
\mathrm{C}_{26} \mathrm{H}_{22} \mathrm{O} \text { (350.5) Ber. C } 89.11 \text { H } 6.33 \text { Gef. C } 89.04 \text { H } 6.50
$$

4. Umlagerung von 11 und 13 zu 10: a) Eine Lösung von $300 \mathrm{mg}(0.86 \mathrm{mmol}) 13 \mathrm{in} 5 \mathrm{ml}$ Methylenchlorid wurde bei $-78^{\circ} \mathrm{C}$ zu $95 \mathrm{mg}(0.95 \mathrm{mmol}) \mathrm{FSO}_{3} \mathrm{H}$, gelöst in ca. $1 \mathrm{ml}$ Methylenchlorid, 
langsam unter Rühren zugetropft. Man erhielt eine dunkelgrüne Lösung. Das Kühlbad wurde entfernt. Nach ca. 10 min erfolgte Farbumschlag nach Rot, und das Reaktionsgemisch wurde in eine auf $-10^{\circ} \mathrm{C}$ vorgekühlte 20 proz. wäßr. KOH-Lösung eingegossen, die organische Phase abgetrennt, mit $10 \mathrm{ml}$ dest. Wasser gewaschen, über $\mathrm{Na}_{2} \mathrm{SO}_{4}$ getrocknet und über $5 \mathrm{~g}$ Kieselgel filtriert (Elutionsmittel Methylenchlorid). Nach Abziehen des Lösungsmittels blieben $266 \mathrm{mg}$ (88\%) 10 als rötliches Harz, das durch Zusatz von wenig Ether zur Kristallisation gebracht werden konnte.

b) In gleicher Weise erhielt man 10 aus 11 mit $65 \%$ Ausbeute.

5. Erzeugung der Carbokationen 3 und 17: a) $205 \mathrm{mg}$ ( $0.59 \mathrm{mmol})$ fein pulverisiertes 8 a wurden bei $-78^{\circ} \mathrm{C}$ unter starkem Rühren spatelweise in eine Lösung von $68 \mathrm{mg}(0.68 \mathrm{mmol}) \mathrm{FSO}_{3} \mathrm{H}$ in $2 \mathrm{ml} \mathrm{SO} 2$ eingestreut. Dabei entsteht eine tiefrote Lösung, deren NMR-Spektren registriert werden können (3): ${ }^{1} \mathrm{H}-\mathrm{NMR}\left(\mathrm{SO}_{2},-70^{\circ} \mathrm{C}\right): \delta($ ext. TMS) $=2.98-3.40(\mathrm{~m} ; 2 \mathrm{H}, 4 \mathrm{a}-\mathrm{H}, 4 \mathrm{~b}-\mathrm{H}), 4.43$ $\left(\mathrm{td}, J_{1,5}=J_{5,4-\text { cis }}=9.5 \mathrm{~Hz}, J_{5,4 \text { trans }}=4.5 \mathrm{~Hz} ; 1 \mathrm{H}, 5-\mathrm{H}\right), 4.69(\mathrm{mc} ; 1 \mathrm{H}, 1-\mathrm{H}), 5.63-6.16(\mathrm{~m}$; $2 \mathrm{H}, 2-\mathrm{H}, 3-\mathrm{H}), 7.0-8.1\left(\mathrm{~m} ; 15 \mathrm{H}\right.$, Aromaten-H). $-{ }^{13} \mathrm{C}-\mathrm{NMR}\left(\mathrm{CDCl}_{3},-50^{\circ} \mathrm{C}\right): \delta=37.3(\mathrm{t}$; C-4), 49.8 (d; C-1), 55.8 (d; C-5), $124.0-139.9$ (Aromaten-C), 152.1 (s; C-7), 182.7 (s; C-6), $210.0\left(\mathrm{~s} ; \mathrm{Ph}_{2} \mathrm{C}\right)$.

b) Dieselben Spektren erhielt man, als $1^{1)}(412 \mathrm{mg}=1.18 \mathrm{mmol})$ in gleicher Weise wie oben mit $137 \mathrm{mg}$ (1.37 mmol) $\mathrm{FSO}_{3} \mathrm{H}$ versetzt wurde. Diese Lösung wurde auf $60 \mathrm{ml} 20 \mathrm{proz}$. wäßr. $\mathrm{K}_{2} \mathrm{CO}_{3}$-Lösung gegossen und das dabei erhaltene organische Material durch PSC (Kieselgel, $\mathrm{CH}_{2} \mathrm{Cl}_{2}$ : Petrolether = 2:1) getrennt. Man isolierte $37 \mathrm{mg} \mathrm{(9 \% )} 8 \mathrm{a}$ und $131 \mathrm{mg}(32 \%) 8 \mathrm{~b}$.

c) $100 \mathrm{mg}(0.29 \mathrm{mmol}) 9,11,12$ oder 13 wurden in $1.5 \mathrm{ml} \mathrm{CDCl}$ gelöst und bei $-78^{\circ} \mathrm{C}$ unter

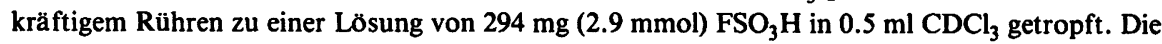
Farbe der zunächst dunkelgrünen Lösung schlägt beim anschließenden Aufwärmen auf $30^{\circ} \mathrm{C}$ nach Rot um. Die davon registrierten ${ }^{1} \mathrm{H}$ - und ${ }^{13} \mathrm{C}$-NMR-Spektren finden sich in Abb. 2 und Tab. 1.

1) E. Bäuml und $H$. Mayr, Chem. Ber. 118, 683 (1985), vorstehend.

2) H. Mayr und $I$. K. Halberstadt-Kausch, Chem. Ber. 115, 3479 (1982).

3) 3a) $K$. Griesbaum, Angew. Chem. 81, 966 (1969); Angew. Chem., Int. Ed. Engl. 8, 933 (1969). 3b) G. Hammen und M. Hanack, Angew. Chem. 91, 649 (1979); Angew. Chem., Int. Ed. Engl. 18, 614 (1979).

4) T. L. Jacobs und D. M. Fenton, J. Org. Chem. 30, 1808 (1965).

5) J. R. Beattier, T. Gilson, K. Livingston, V. Fawcett und G. A. Ozin, J. Chem. Soc. A 1967, 712.

6) H. M. McConnell und R. E. Robertson, J. Chem. Phys. 29, 1361 (1958).

7) L. R. Schmitz und T. S. Sorensen, J. Am. Chem. Soc. 104, 2600 (1982).

8) 8a) G. A. Olah, G. Asensio und H. Mayr, J. Org. Chem. 43, $1518(1978)$. - 8b) N. C. Deno, C. U. Pittman jr. und J. O. Turner, J. Am. Chem. Soc. 87, 2153 (1965). - 8c) C. U. Pittman und W. G. Miller, J. Am. Chem. Soc. 95, 2947 (1973).

9) Vorläufige Mitteilung: $H$. Mayr und $E$. Bauml, Tetrahedron Lett. 24, 357 (1983).

10) Wilhelm von Occam, $1285-1349$.

11) H. Saimoto, C. Houge, A.-M. Hesbain-Frisque, A. Mockel und L. Ghosez, Tetrahedron Lett. 24, 2251 (1983).

12) R. B. Woodward und R. Hoffmann, Angew. Chem. 81, 797 (1969); Angew. Chem., Int. Ed. Engl. 8, 781 (1969). 